\title{
Extracorporeal Life Support and New Therapeutic Strategies for Cardiac Arrest Caused by Acute Myocardial Infarction - a Critical Approach for a Critical Condition
}

\author{
Theodora Benedek ${ }^{1^{*}}$, Monica Marton Popovici ${ }^{2}$, Dietmar Glogar ${ }^{3}$ \\ 1 University of Medicine and Pharmacy Tirgu Mures, Romania, Clinic of Cardiology \\ 2 Swedish Medical Center, Department of Internal Medicine and Critical Care, Edmonds, Washington, USA \\ 3 Medical University of Vienna, Austria
}

\begin{abstract}
This review summarizes the most recent developments in providing advanced supportive measures for cardiopulmonary resuscitation, and the results obtained using these new therapies in patients with cardiac arrest caused by acute myocardial infarction (AMI). Also detailed are new approaches such as extracorporeal cardiopulmonary resuscitation (ECPR), intra-arrest percutaneous coronary intervention, or the regional models for systems of care aiming to reduce the critical times from cardiac arrest to initiation of ECPR and coronary revascularization.
\end{abstract}

Keywords: cardiac arrest, intra-arrest $\mathrm{PCl}$, cardiopulmonary resuscitation

Received: 05 August 2016 / Accepted: 20 September 2016

\section{INTRODUCTION}

Acute myocardial infarction (AMI) continues to represent a major issue for the healthcare system, being associated with a high mortality rate. In spite of the introduction of new therapies for acute coronary syndromes (ACS), the mortality associated with ACS remains as high as $20-30 \%$ [1]. Newer treatments, from primary percutaneous interventions in the emergency settings to therapeutic hypothermia in the post-resuscitation care and stem cell therapy in the post-infarction period, have significantly improved the prognosis of these patients [2,3]. Primary percutaneous intervention (PCI), performed within twelve hours from the onset of symptoms, has been demonstrated to represent the most efficient therapy for recanalization of obstructed coronary arteries and to decrease the AMI-related mortality to $3-5 \%$ in high-performance centers [4]. However, PCI can be performed only in patients who arrive in time in the catheterization laboratory. One of the major complications of AMI is represented by cardiac arrest, that can occur at home, immediately after the onset of symptoms, and before the patient is taken to a PCI center.

Sudden cardiac arrest (CA) is associated with extremely high mortality rates, which have remained high despite the major progress in the development of new therapies. The outcome for patients suffering an out of hospital cardiac arrest (OHCA) remains poor compared to patients with in-hospital cardiac arrest (IHCA), the latter having the advantage of rapid initiation of cardiopulmonary resuscitation (CPR) and early access to advanced support therapies $[5,6]$.

Despite the significant recent progress in decreasing both the mortality and the rate of neurological alterations following CA, the survival rate after CA remains poor. Therefore, new advanced supportive strategies

\footnotetext{
Correspondence to: Theodora Benedek: University of Medicine and Pharmacy Tirgu Mures, Romania, Gheorghe Marinescu street no.50, 540139 Tirgu Mures, Romania. Tel-Fax +40265211595. E-mail: theodora.benedek@gmail.com

Monica Marton-Popovici, Swedish Medical Center, Department of Internal Medicine and Critical Care, 21601, 76th Ave W, Edmonds, Washington, 98026, USA. E-mail: Monica.MartonPopovici@Swedish.org

Dietmar Glogar: Medizinische Universität Wien Univ. Klinik für Innere Medizin II Abteilung für Kardiologie Waehringer Guertel 18-20, A-1090 Wien, Austria. E-mail: d.glogar@aon.at
} 
are required to further improve the success rates following resuscitation for CA in all patient categories, including AMI patients.

New supportive strategies include the use of devices for mechanical chest compression (mechanical resuscitation), that provides assistance for a more effective resuscitation in cases with prolonged duration, and devices for extracorporeal cardiopulmonary resuscitation (ECPR), that ensure the maintenance of circulation and perfusion of vital organs.

This review summarizes the most recent developments in providing advanced supportive measures for cardiopulmonary resuscitation, and the results obtained using these new therapies in patients with cardiac arrest caused by AMI. Also, new approaches for AMI patients are presented such as the concept of ECPR, intra-arrest percutaneous coronary intervention, or the regional models for systems of care aiming to reduce the critical times from cardiac arrest to initiation of ECPR and coronary revascularization.

\section{DXTRACORPOREAL LIFE SUPPORT IN CRITICAL CARE}

\section{Extracorporeal life support - a therapeutic alternative}

The Extracorporeal life support (ECLS) was first suggested in 1976 as a therapeutic alternative for refractory cases of cardiac arrest unresponsive to CPR [7-11]. The ECLS is used at present as an adjuvant therapy for conventional $\mathrm{CPR}$, to increase the survival rate post-CPR. Table 1 summarizes the survival rates associated with ECPR reported by different clinical studies in various clinical settings. In a recent study, survival and neurologic outcomes were superior in ECPR group at 30 days after resuscitation as compared to conventional CPR [12].

The concept of ECPR is based on utilization of an extracorporeal membrane oxygenation (ECMO) system, which includes a membrane oxygenator connected to a centrifugal pump and a heat exchanger, interconnected via a bypass which is inserted percutaneously into the femoral artery and vein [13]. In patients with shock or cardiac arrest, insertion of such a veno-arterial extracorporeal membrane oxygenation system can be extremely useful in providing rapid circulatorysupport, and medical staff should be trained to set up and initiate the ECMO device within ten minutes [13].

Recent progress in medical technology has led to a significant reduction in the size of the devices used for ECLS, making it possible to bring the device to the patient place in cases of out-of-hospital cardiac arrest (OHCA) [13]. However, the results encountered when using ECLS in OHCA patients continue to be inferior to those recorded in patients with in-hospital cardiac arrest (IHCA) [14].

ECPR was considered an attractive concept for treating patients with IHCA, in whom the results obtained using ECLS are significantly superior to those obtained using conventional CPR. Several studies have reported that ECPR results in haemodynamic stabilization, increased rate of return of spontaneous circulation (ROSC), improved neurologic outcomes and improved survival compared to conventional CPR [12]. However, it is important to remember that ECPR only ensures the circulatory support for critical cases, providing an adequate blood flow to vital organs before a ROSC is achieved; it does not treat the disease which led to cardiac arrest [15].

\section{ECPR- who, when and to whom?}

A survey including 70 centers performing ECPR indicated that cannulation is usually performed by cardiothoracic surgeons while almost all interventions are conducted by a mixed interdisciplinary team including critical care specialists, surgeons, perfusionists, and pharmacists. This survey also indicated that most of the centers do not have a well-defined list of inclusion/ex-

Table 1. ECPR survival rates reported in clinical studies

\begin{tabular}{|c|c|c|c|c|}
\hline Author & Patient population & $\mathbf{n}$ & Survival rate & Endpoint \\
\hline Kim et al [43] & $\mathrm{OHCA}$ & 55 & $30 \%$ & Survival to discharge \\
\hline Beurtheret et al [56] & Refractory CS & 104 & $36.8 \%$ & Survival to discharge \\
\hline Yamada et al [54] & OHCA & 688 & $5.9 \%$ & 90-days survival rate \\
\hline Kagawa et al [52-52] & Intra-arrest $\mathrm{PCl}$ and ECPR & 86 & $36 \%$ & Survival to discharge. \\
\hline Yannopoulos et al [67] & OHCA & 27 & $55 \%$ & Survival to discharge \\
\hline Sharma et al [66] & $\mathrm{OHCA}$ & 195 & $46.2 \%$ & Survival to discharge \\
\hline Le Guen et al [7] & OHCA and refractory CA & 51 & $4 \%$ & 30-days survival \\
\hline
\end{tabular}


clusion criteria when selecting a patient for ECPR [16]. Generally, it is recommended that the interdisciplinary team should be activated in order to decide whether or not to initiate ECPR in patients who do not present with any sign of a return of spontaneous circulation (ROSC) after ten minutes of advanced cardiac life support [17].

According to the current recommendations, ECPR is indicated in cases when vital signs are still present during CPR or when cardiac arrest is secondary to intoxication or hypothermia, in patients with no-flow duration less than five minutes and when the time from conventional $\mathrm{CPR}$ to ECPR is less than one hundred minutes [18].

Several of the criteria proposed for including a patient in an ECPR program are: ventricular fibrillation recorded on the initial electrocardiogram, a time interval of fewer than fifteen minutes from cardiac arrest until the initiation of resuscitation, presumed ischemic aetiology, and ROSC not achieved within twenty minutes of the initiation of conventional CPR $[13,14]$.

However, the current guidelines state that there is insufficient evidence to recommend routine use of ECPR in patients with CA, this method being indicated mainly in cases when the suspected aetiology is potentially reversible after the cardiorespiratory support, but only in those settings when this can be rapidly implemented [19].

\section{Ethical issues related to ECPR}

The ethical issues associated with the use of ECPR were recently addressed in several publications which concluded that ECPR should be considered first, before the commencement of any organ donation procedure, due the risk of including a patient in an organ donation protocol when the patient might have been saved by the use of ECPR [20,21].

\section{In-hospital vs. out of hospital ECPR}

There is growing evidence that ECPR could be beneficial especially for IHCA patients while the results are less favorable for patients who develop CA out of a hospital setting [22]. Several studies demonstrated the ECPR is associated with significantly better results in in-hospital CPR than in out-of-hospital CA [6,23-32]. This is probably due to the faster decision time and the more rapid initiation of ECPR, following the decision in IHCA patients.

In a meta-analysis, including 38.160 patients and neurological outcomes as endpoints, ECPR showed significantly better results than conventional CPR for inhospital patients, while for out-of-hospital CA patients the difference between ECPR and standard CPR was not statistically significant [33]. In another study on 955 patients with in-hospital or out-of-hospital CPR, IHCA patients presented a significantly higher rate of survival discharge as compared to OHCA patients $(p<0.0001)$ [34]. In a 5-years long study of ECPR for refractory OHCA, the percentage of patients who were successfully weaned from the system reached $11.7 \%$ while only $8.8 \%$ of patients survived to discharge [35]. All these data indicate that ECPR represents a viable alternative especially in critical cases when CA occurs in hospitalized patients.

\section{Neurological outcomes after ECPR}

Neurological outcomes after CA are key determinants of the future prognosis and quality of life in these critical patients [35-40]. In a study on 171 patients refractory to conventional CPR who underwent emergency cardiopulmonary bypass, insertion of intra-aortic counterpulsation pump and PCI, induction of therapeutic hypothermia at $34^{\circ} \mathrm{C}$ for 3 days, a collapse-tobypass time of 55.5 minutes and a bypass-to- $34^{\circ} \mathrm{C}$ of 21.5 minutes were the cut off levels for predicting a favorable neurologic outcome [41].

In a small study on 24 patients with refractory CA treated with ECPR, satisfactory rates of survival good neurologic recovery were achieved in both IHCA and OHCA patients [8]. At the same time, this technique leads to rapid stabilization of the haemodynamic status in these critical patients [8,42].

In a larger study on 499 patients with CA, the transfer from conventional CPR to ECPR was associated with a better neurological outcome in patients with a duration of $\mathrm{CPR}>21$ minutes, especially in younger patients, who presented without initial asystole [43]. This study demonstrated a more favorable three months neurological outcome in patients receiving ECPR than in those receiving conventional CPR when the duration of CPR was $>21$ minutes $(\mathrm{p}<0.0001)$. However, it is important to note that the group showing a good neurologic outcome presented a higher incidence of therapeutic hypothermia, healthy coronary arteries and lower ECPR-associated complication rates [43].

\section{CARDIAC ARREST IN PATIENT WITH ACUTE MYOCARDIAL INFARCTION}

Acute myocardial infarction is produced by the sudden occlusion of a coronary artery, usually resulting from 
the rupture or erosion of a vulnerable atheromatous coronary plaque [44]. Plaque rupture or erosion often occurs at luminal sites where the percentage of coronary narrowing, as determined by coronary angiography, is not high. In $40 \%$ of victims of sudden coronary death, autopsy studies identified the location of plaque rupture at the level of a coronary lesion producing less than 50 percent diameter stenosis [45]. As a luminal narrowing of less than $50 \%$ can remain clinically silent until the moment the plaque ruptures and the subsequent formation of an intracoronary thrombosis, many of these patients can remain symptoms-free until the onset of the infarction. In such circumstances, CA can represent the first manifestation of coronary atherosclerosis and in most of these cases CA occurs out of a hospital setting. This scenario is associated with worst prognosis due to the delay in initiating appropriate supportive measures and CPR.

A recent study indicated that the primary factor associated with resistance to conventional CPR and the need to undertake ECPR was myocardial ischemia. In this study, patients who required ECPR presented an $87 \%$ rate of non-recanalization during coronary angiography compared to $58 \%$ in the nonECPR group $(\mathrm{p}=0.03)[46]$.

\section{Complex ECPR-related approaches in CA from pre- sumed ischemic origin}

A comprehensive approach in patients with reversible CA caused by acute myocardial infarction includes performance of percutaneous coronary interventions, therapeutic hypothermia to ensure neuroprotection and maintenance of organ function until recovery $[47,48]$.

The CHEER trial studied a complex approach including mechanical CPR, ECMO initiated by critical care specialists by femoral vein and artery cannulation, therapeutic hypothermia at $33^{\circ} \mathrm{C}$ for 24 hours and early reperfusion of occluded coronary arteries in patients with cardiac arrest. This trial demonstrated that a protocol instituted by critical care specialists that include ECPR, mechanical CPR, and therapeutic hypothermia is associated with a $54 \%$ survival rate with full neurologic recovery in patients with CA refractory to conventional CPR [49].

Prague OHCA study aimed to demonstrate the role of a hyper-invasive approach, including mechanical chest compression devices, cooling, ECLS and an early invasive revascularization approach, in patients with
OHCA of presumed cardiac origin [50]. The preliminary results of this study showed encouraging data, with a survival rate of $20 \%$ and a cerebral performance category of 1 and 2 in $75 \%$ of survivors. However, the survival rate in patients admitted under ongoing CPR was only $30 \%$ [50].

In patients with CA caused by AMI, the post-resuscitation care should be carefully considered, including advanced measures including both reperfusion therapy and therapeutic hypothermia. When a patient undergoing ECPR is haemodynamically unstable, adjunctive steps should be rapidly initiated, including transfer to the catheterization laboratory, intra-aortic balloon counterpulsation or hypothermia induction by rapid injection of cold saline [51,52].

In OHCA patients with ventricular tachycardia/ventricular fibrillation as shown on the initial electrocardiogram, a complex treatment including ECPR, therapeutic hypothermia, and insertion of an intra-aortic balloon pump significantly improved neurological outcomes. However, in patients without pre-hospital return of spontaneous circulation, implementation of ECPR did not lead to a significantly better result [53].

In the CRITICAL registry, a Japanese network of eleven critical care centers enrolled 688 consecutive patients with OHCA, out of which only $3.0 \%$ received ECPR. This study aimed to provide the most appropriate therapeutic strategy, based on a mixed approach including PCI, cooling and ECPR for out-of-hospital cardiac arrest patients with presumed ischemic origin, preliminary data indicating very low rates of survival at 90-day and neurological performance $(5.9 \%$ and $3.0 \%$ respectively) [54].

\section{Intra-arrest percutaneous coronary intervention}

Only very few observational studies addressed the role of primary PCI in CA patients, indicating that a combined approach including ECPR and PCI may improve the outcomes of patients with refractory CA $[51,52,55]$.

In a study including 86 patients with ACS unresponsive to conventional CPR, intra-arrest PCI was performed in $71 \%$ of the cases, leading to the return of spontaneous heart beat in all the cases. In those who survived 30 days, the rate of intra-arrest PCI was higher $(\mathrm{p}=0.04)$ and the time interval from CA to initiation of ECPR was shorter $(\mathrm{p}=0.002)$. In this study, insertion of an intra-aortic balloon pump was undertaken in $83 \%$ of cases while mild therapeutic hypothermia was indicated in only $37 \%$ of cases. Patients who received 
intra-arrest PCI had more favorable outcomes, such as a superior ROSC rate $(\mathrm{p}<0.001)$, a higher percentage of weaning from ECMO ( $\mathrm{p}=0.009)$, a superior 30 -days survival rate $(\mathrm{p}=0.03)$ and better neurological outcomes $(\mathrm{p}=0.005)$ [51,52]. This study demonstrated that intra-arrest PCI plus ECPR could decrease mortality in cardiac arrest patients who are unresponsive to CPR.

\section{ECPR in acute myocardial infarction with cardiogenic shock}

In the setting of an AMI, cardiogenic shock is associated with high mortality when emergency circulatory support is not initiated in a timely and efficient manner [56,57]. In these critical cases, ECPR can serve as a bridge to transplantation, to implantation of a longterm assist device or to recovery, while insertion of an intra-aortic balloon pump can provide additional haemodynamic support during the bridging period.

In the case of cardiogenic shock and acute myocardial infarction, transportation of the patient to a highly specialized tertiary center is the key to survival. In the Cardiac-RESCUE program, eighty-seven consecutive patients with OHCA and refractory cardiogenic shock received ECPR support followed by emergency transport to a tertiary center. Thirty-two patients $(36.8 \%)$ survived to hospital discharge, and the most powerful independent predictor of mortality was the initiation of ECPR $(p<0.0001)$. Interestingly, after adjusting for confounding factors, the survival rate was not significantly different between cardiogenic shock patients with OHCA and those with IHCA [56].

\section{DYSTEMS OF CARE FOR CARDIAC ARREST FOLLOWING ACUTE MYOCARDIAL INFARCTION}

\section{Regional models for implementation of ECPR}

Various regional models have been proposed to implement the utilization of ECPR in CA patients, in a similar fashion to the implementation of the ST-elevation myocardial infarction (STEMI) networks, which proved to be extremely efficient in reducing STEMIrelated mortality $[4,58,59]$.

Implementation of new therapeutic approaches in the community can lead to significant improvement in the rate of survival accompanied by good neurological outcomes $[60,61]$. For instance, implementation of a system-wide approach to OHCA patients, including therapeutic hypothermia for comatose patients, initiation of ECPR, immediate coronary angiography in cases with presumed ischemic etiology of the cardiac arrest and urgent defibrillation at pre-hospital and hospital level increased the rate of discharge with good neurologic outcomes from 3.3\% in 2009 to $8.5 \%$ in 2013 $(\mathrm{p}<0.001)$ [62]. Also, another study on 1128 OHCA patients enrolled between 2007 and 2011, showed that OHCA survival was significantly improved, from 5.6\% to $13.01 \%$, by the implementation of new approaches, consisting in automated external defibrillators, therapeutic hypothermia and implementation of ECPR programs for selected cases [63].

A Canadian registry on consecutive adults with non-traumatic OHCA showed that the integration of an ECPR program into an existing high-performance system of care might have a significant impact on patients outcome, with $68 \%$ of OHCA patients surviving to hospital admission and $42 \%$ surviving to hospital discharge [64,65].

A study of ECPR utilization for OHCA patients in the Maastricht region of southeast Netherlands analyzed the outcome of CA patients after implementing ECPR methods and proved that a shockable rhythm at presentation, the presence of ROSC and appropriate post-arrest care could improve the results of CA patients. In this study, the survivors' group presented more frequently with ACS ( $\mathrm{p}=0.01$ ), and benefitted to a greater extend from therapeutic hypothermia and PCI $(p<0.01)$ [66]. In this network, average critical times encountered were 7 minutes for EMS response time, 24 minutes for the duration of advanced cardiac life support and 12 minutes for the transport to the emergency department [66].

In the Minessota study, including three emergent medical services systems which performed automated $\mathrm{CPR}$ and transport to an University hospital where EMCO was initiated, followed by coronary angiography and PCI, $78 \%$ of patients survived to hospital admission, and 55\% survived to hospital discharge, while $50 \%$ achieved good neurologic outcomes [67]. However, it is important to note that in this study patients also received therapeutic hypothermia.

In another study, the increase in prehospital duration of CPR was associated with adverse outcomes despite the introduction of ECPR, indicating that transportation to a more definitive treatment facility should be preferred rather than pre-hospital initiation of ECPR in cases with an extended CPR duration [68]. 


\section{Critical time intervals in the ECPR networks}

Similarly to the terms "door to needle" and "door to balloon" that are currently used to characterize the efficiency of a hospital-based system for treating myocardial infarction, the term "door-to-implantation" was proposed for patients with CA, to describe the time from arrival of the patient in a hospital to the time of implantation of the ECMO system in the catheterization laboratory, during PCI.

The studies demonstrated that such a "door-to-implantation" time less than thirty minutes significantly improved the survival rate at thirty days, in patients with OHCA. This time was significantly higher in non-survivors than in survivors $(\mathrm{p}<0.05)$, being the most powerful predictor of mortality in these critical patients [69].

The ECPR was significantly more efficient when bystander CPR had been initiated and when initiation of ECPR was preceded by a short period of conventional CPR [70].

A small study of 35 patients who received ECMO failed to identify any significant difference between survivors and non-survivors regarding the time of arrest to CPR ( $\mathrm{p}=0.4)$ and the time of CPR to ECMO ( $\mathrm{p}=0.5)$, while the survivor group received in a significantly larger proportion coronary revascularization $(p=0.02)$ [27]. However, a longer time interval from cardiac arrest to initiation of extracorporeal life support demonstrated to be associated with higher mortality rates [71].

The optimum time intervals for on-scene resuscitation have been addressed in a large study of 6571 cases, in which ROC analysis demonstrated that a cut off of sixteen minutes of resuscitation represented a good predictor of a favourable outcome. This study recommended a transport time of 8 to 24 minutes to be considered as the limits within which on-scene resuscitation should be performed [64].

\section{WEWER DEVELOPMENTS IN ADVANCED SUPPORTING MEASURES FOR CPR}

\section{Mechanical resuscitation systems}

Automated chest compression systems have been introduced as adjunctive measures in cases necessitating CPR, in an attempt to assist the rescuers in their efforts to provide consistent and sustained efficient compressions [72].

In patients with CA of presumed ischemic origin, mechanical chest compression systems have been de- veloped to serve as a bridge to a better-quality support system such as ECPR or during transportation to the catheterization laboratory. Such devices increase regional cerebral oxygenation during resuscitation and improve neurological outcomes in successfully resuscitated patients with cardiac arrest.

Experimental data on porcine models proved that automatic chest compression does not interfere with extracorporeal life support systems that can be successfully installed during chest compression [73]. In an extensive study on 4868 OHCA patients out of which 285 received automated mechanical compression with the use of an automated external chest compression device, the device proved to be easy to use, being installed on average in thirty seconds [74].

Several new devices have been introduced to improve the $\mathrm{SO} 2$ and the neurological outcomes following CPR, such as load band distributing CPR. These have been shown to increase $\mathrm{SO} 2$ compared with the conventional technique [75]. In several countries which have developed a nationwide program for organ harvesting, all the emergency units have been equipped with devices allowing automated chest compression [7]. However, there is insufficient data to support or reject the use of these devices for mechanically assisted $\mathrm{CPR}$, their validation being based mainly on observational studies [76].

\section{Therapeutic hypothermia and ECPR}

Despite the great expectations of this therapeutic approach, the current evidence indicated no benefit from infusion of cold saline solutions in the pre-hospital setting [19,77]. Current guidelines do not recommend the routine pre-hospital cooling of patients when spontaneous circulation is established and suggest that this should remain reserved only for well-defined cases. However, the addition of therapeutic hypothermia to multiple protocols for CPR can lead to better mortality and neurological outcomes. According to the CHEER trial, a complex protocol that includes therapeutic hypothermia and ECPR results in a survival rate of $54 \%$ associated with full neurologic recovery [49]. Moreover, the addition of therapeutic hypothermia and early haemodynamic stabilization improved the neurological outcomes in patients with resuscitated CA [43].

\section{DREDICTORS OF SURVIVAL IN ECPR}

Factors influencing the outcome after CPR include the duration of CPR, the initial cardiac rhythm, the etiol- 
Table 2 - Predictors of survival in ECPR

\begin{tabular}{lll} 
Author & Predictor & Characteristic \\
Lee et al [34], Haneya et al [79] & Initial cardiac rhythm & Shockable cardiac rhythm \\
\hline Lee et al [34], Haneya et al [79] & Duration of CPR & $\begin{array}{l}\text { For every 1- minute increase in CPR duration, survival } \\
\text { decreases by 4\% }\end{array}$ \\
\hline Le Guen et al [7] & Delay to initiate ECPR & $>10$ minutes \\
\hline Le Guen et al [7] & Duration of no-flow & $<5$ minutes \\
\hline Lee et al [34], Haneya et al [79] & Etiology of cardiac arrest & Non-ischemic etiology \\
\hline Flecher et al [83] & Age & $<65$ years \\
\hline Lee et al [34] & IHCA & IHCA presented 2.49 better survival rate than OHCA \\
\hline Lee et al [12] & Oliguria & $>0.5$ mL/kg/h in the first 24 h \\
\hline Flecher et al [83] & Serum lactate & $<12.0 \mathrm{mM} / \mathrm{l}$ \\
\hline Anselmi et al82 & Duration of ECPR & $>30$ min \\
\hline Flecher et al [83] & pH & $>7$ \\
\hline Anselmi et al82 & Body temperature & Low body temperature at the time of ECPR implantation \\
\hline *HCA out-of-hospital cardiac arrest, IHCA = in-hospital cardiac arrest, CPR = cardiopulmonary resuscitation, ECPR=extracorporeal cardiopulmonary resuscitation
\end{tabular}

ogy of cardiac arrest, the underlying disease, or age [34,78-80]. At the same time, ACS and OHCA were significant negative risk factors for ROSC, with a ROSC rate 0.63 times lower in the ACS group as compared to the non-ACS group. The most significant predictors of survival in patients undergoing ECPR for CA are listed in Table 2.

In another retrospective study, the thirty days survival rate for OHCA was 43.5\% when ECMO was initiated within ten minutes [34]. Important to note is the fact that with every one minute increase in CPR duration there is a $4 \%$.decrease in the survival rate [34].

According to a small study of fifty-one cases with refractory cardiac arrest, factors related to low survival rates in ECPR were the delay in initiating ECPR and the no-flow duration [7]. Several studies demonstrated that oliguria during the first twenty-four hours after ECMO represented an independent predictor of mortality [12]. In another predictive model, a higher serum lactate level, an increased duration of ECPR and a low body temperature at the time of ECPR implantation, were predictors of mortality in cardiac arrest patients receiving ECPR [81,82].

\section{CONCLUSIONS}

The survival of patients with cardiac arrest caused by acute myocardial infarction is largely dependent on the proper implementation of adequate therapeutic protocols to provide adequate circulatory support. ECPR proved to be associated with a significant decrease in mortality when performed timeously in well-selected cases.
In patients with OHCA caused by acute myocardial infarction, the key factors for a higher survival rate are a well-functioning prehospital emergency service, able to provide ECPR within several minutes and the transportation of the patient to well-qualified centers for urgent PCI.

A multidisciplinary team including cardiologists, heart failure specialists, critical care and neuro-critical care specialists should be involved in this therapeutic algorithm to ensure the proper management of these critical cases.

\section{DISCLOSURE OF CONFLICT OF INTEREST}

Nothing to declare

\section{REFERENCES}

1. Chen HY, Gore JM, Lapane KL, et al. A 35-Year Perspective (1975 to 2009) into the Long-Term Prognosis and Hospital Management of Patients Discharged from the Hospital After a First Acute Myocardial Infarction. Am J Cardiol. 2015;116:24-9. doi: 10.1016/j.amjcard.2015.03.035.

2. Islam S, Hampton-Till J, Mohd Nazri S, et al. Setting Up an Efficient Therapeutic Hypothermia Team in Conscious ST Elevation Myocardial Infarction Patients: A UK Heart Attack Center Experience. Ther Hypothermia Temp Manag. 2015;5:217-22. doi: 10.1089/ther.2015.0012.

3. Gyongyosi M, Hemetsberger R, Posa A, et al. Hypoxiainducible Factor 1-Alp[ha Release After Intracoronary versus Intramyocardial Stem Cell Therapy in Myocardial Infarction. J Cardiovasc Translational Res. 2010;3:114-21. doi: 10.1007/ s12265-009-9154-1.

4. Benedek I, Gyongyosi M, Benedek T. A prospective regional 
registry of ST-elevation myocardial infarction in central Romania. Impact of the Stent for Life initiative recommendations on patient outcomes. Am Heart J. 2013;166:457-65. doi: 10.1016/j.ahj.2013.03.033.

5. Avalli L, Maggioni E, Formica F, et al. Favourable survival of inhospital compared to out-of-hospital refractory cardiac arrest patients treated with extracorporeal membrane oxygenation: an Italian tertiary care centre experience. Resuscitation. 2012;83:579-83. doi: 10.1016/j.resuscitation.2011.10.013.

6. Dworschak M. Is extracorporeal cardiopulmonary resuscitation for out-of-hospital cardiac arrest superior compared with conventional resuscitation? Crit Care Med. 2013;41:1365-6. doi: 10.1097/CCM.0b013e31828044c0.

7. Le Guen M, Nicolas-Robin A, Carreira S, et al. Extracorporeal life support following out-of-hospital refractory cardiac arrest. Crit Care. 2011;15:R29. doi: 10.1186/cc9976.

8. Fagnoul D, Taccone FS, Belhaj A, et al. Extracorporeal life support associated with hypothermia and normoxemia in refractory cardiac arrest. Resuscitation. 2013;84:1519-24. doi: 10.1016/j.resuscitation.2013.06.016.

9. Lamhaut L, Jouffroy R, Kalpodjian A, et al. Successful treatment of refractory cardiac arrest by emergency physicians using pre-hospital ECLS. Resuscitation. 2012;83:e177-e178. doi: 10.1016/j.resuscitation.2012.03.016

10. Mojoli F, Venti A, Pellegrini $C$, et al. Hospital survival and longterm quality of life after emergency institution of venoarterial ECMO for refractory circulatory collapse. Minerva Anestesiol. 2013;79:1147-55.

11. Wang $\mathrm{CH}$, Chen YS, Ma MH. Extracorporeal life support. Curr Opin Crit Care. 2013;19:202-07. doi: 10.1097/ MCC.0b013e32836092a1.

12. Lee JJ, Han SJ, Kim HS, et al. Out-of-hospital cardiac arrest patients treated with cardiopulmonary resuscitation using extracorporeal membrane oxygenation: focus on survival rate and neurologic outcome. Scand J Trauma Resusc Emerg Med. 2016;24:74. doi: 10.1186/s13049-016-0266-8.

13. Kagawa E. Extracorporeal cardiopulmonary resuscitation for adult cardiac arrest patients. World J Crit Care Med. 2012;1:469. doi: 10.5492/wjccm.v1.i2.46.

14. Kagawa E, Inoue I, Kawagoe T, et al. Assessment of outcomes and differences between in- and out-of-hospital cardiac arrest patients treated with cardiopulmonary resuscitation using extracorporeal life support. Resuscitation. 2010;81:968-73. doi: 10.1016/j.resuscitation.2010.03.037.

15. Patroniti N, Sangalli F, Avalli L. Post-cardiac arrest extracorporeal life support. Best Pract Res Clin Anaesthesiol. 2015;29:497-508. doi: 10.1016/j.bpa.2015.09.004.

16. Tonna JE, Johnson NJ, Greenwood J, et al. Practice characteristics of Emergency Department extracorporeal cardiopulmonary resuscitation (eCPR) programs in the United States: The current state of the art of Emergency Department extracorporeal membrane oxygenation (ED ECMO). Resuscitation. 2016;107:38-46. doi: 10.1016/j.resuscitation.2016.07.237.
17. Teschendorf P, Bernhard M. A bridge to life: ECPR who, when, where and why? Resuscitation. 2014;85:709-10. doi: 10.1016/j. resuscitation. 2014.03.315.

18. Rousse N, Robin E, Juthier F, et al. Extracorporeal Life Support in Out-of-Hospital Refractory Cardiac Arrest. Artif Organs. 2016;40:904-9. doi: 10.1111/aor.12655.

19. Link MS, Berkow LC, Kudenchuk PJ, et al. Part 7: Adult Advanced Cardiovascular Life Support: 2015 American Heart Association Guidelines Update for Cardiopulmonary Resuscitation and Emergency Cardiovascular Care. Circulation. 2015;132 (18Suppl2):S444-64. doi: 10.1161/CIR.0000000000000261.

20. Dalle Ave AL, Shaw DM, Gardiner D. Extracorporeal membrane oxygenation (ECMO) assisted cardiopulmonary resuscitation or uncontrolled donation after the circulatory determination of death following out-of-hospital refractory cardiac arrest - An ethical analysis of an unresolved clinical dilemma. Resuscitation. 2016; pii:S0300-9572(16)30150-2. doi: 10.1016/j.resuscitation.2016.07.003 [Epub ahead of print]

21. Mancini ME, Diekema DS, Hoadley TA, et al. Part 3: Ethical Issues: 2015 American Heart Association Guidelines Update for Cardiopulmonary Resuscitation and Emergency Cardiovascular Care. Circulation. 2015;132(18Suppl2):S383-S396. doi: 10.1161/CIR.0000000000000254.

22. Lin JW, Wang MJ, Yu HY, et al. Comparing the survival between extracorporeal rescue and conventional resuscitation in adult in-hospital cardiac arrests: propensity analysis of threeyear data. Resuscitation. 2010;81:796-803. doi: 10.1016/j. resuscitation.2010.03.002.

23. Biderman P, Kagan I, Jakobishvili Z, Fainblut M, Lishetzinsky Y, Cohen J. Extracorporeal Cardiopulmonary Resuscitation for Out-of-Hospital Cardiac Arrest. Isr Med Assoc J. 2016;18:61-2.

24. Choi DS, Kim T, Ro YS, et al. Extracorporeal life support and survival after out-of-hospital cardiac arrest in a nationwide registry: A propensity score-matched analysis. Resuscitation. 2016;99:26-32. doi: 10.1016/j.resuscitation.2015.11.013.

25. Flannigan C, Bourke TW, Chisakuta A. Out-of-hospital extracorporeallife support-when is itappropriate? Resuscitation. 2012;83:e85. doi: 10.1016/j.resuscitation.2011.08.030.

26. Fukuda T, Nakamura K, Fukuda-Ohashi N, Yahagi N. How long should resuscitative efforts be continued in adult out-ofhospital cardiac arrest? Can J Cardiol. 2015;31:364.e1-2. doi: 10.1016/j.cjca.2014.11.028.

27. Ha TS, Yang JH, Cho YH, et al. Clinical outcomes after rescue extracorporealcardiopulmonaryresuscitationforout-of-hospital cardiac arrest. Emerg Med J. 2016; pii:emermed-2015-204817. doi: 10.1136/emermed-2015-204817 [Epub ahead of print].

28. Maekawa K, Tanno K, Hase M, Mori K, Asai Y. Extracorporeal cardiopulmonary resuscitation for patients with out-of-hospital cardiac arrest of cardiac origin: a propensity-matched study and predictor analysis. Crit Care Med. 2013;41:1186-96. doi: 10.1097/CCM.0b013e31827ca4c8.

29. Putzer G, Mair B, Hangler H, Ströhle M, Mair P. Emergency extracorporeal life support after prolonged out-of-hospital 
cardiac arrest. J Cardiothorac Vasc Anesth. 2014;28:1024-26. doi: 10.1053/j.jvca.2013.12.016.

30. Ortega-Deballon I, Hornby L, Shemie SD, Bhanji F, Guadagno E. Extracorporeal resuscitation for refractory out-of-hospital cardiac arrest in adults: A systematic review of international practices and outcomes. Resuscitation. 2016;101:12-20. doi: 10.1016/j.resuscitation.2016.01.018.

31. Sawyer KN, Kurz MC. Caution when defining prolonged downtime in out of hospital cardiac arrest as extracorporeal cardiopulmonary resuscitation becomes accessible and feasible. Resuscitation. 2014;85:979-80. doi: 10.1016/j. resuscitation.2014.05.018.

32. Wang CH, Chou NK, Becker LB, et al. Improved outcome of extracorporeal cardiopulmonary resuscitation for out-ofhospital cardiac arrest-a comparison with that for extracorporeal rescue for in-hospital cardiac arrest. Resuscitation. 2014;85:1219-24. doi: 10.1016/j.resuscitation.2014.06.022.

33. Ahn C, Kim W, Cho Y, Choi KS, Jang BH, Lim TH. Efficacy of extracorporeal cardiopulmonary resuscitation compared to conventional cardiopulmonary resuscitation for adult cardiac arrest patients: a systematic review and meta-analysis. Sci Rep. 2016;6:34208. doi: 10.1038/srep34208.

34. Lee $\mathrm{YH}, \mathrm{Oh} \mathrm{YT}, \mathrm{Ahn} \mathrm{HC}$, et al. The prognostic value of the grey-to-white matter ratio in cardiac arrest patients treated with extracorporeal membrane oxygenation. Resuscitation. 2016;99:50-5. doi: 10.1016/j.resuscitation.2015.11.009.

35. Lee SH, Jung JS, Lee KH, Kim HJ, Son HS, Sun K. Comparison of Extracorporeal Cardiopulmonary Resuscitation with Conventional Cardiopulmonary Resuscitation: Is Extracorporeal Cardiopulmonary Resuscitation Beneficial? Korean J Thorac Cardiovasc Surg. 2015;48:318-27. doi: 10.5090/ kjtcs.2015.48.5.318.

36. Pozzi M, Koffel C, Armoiry X, et al.Extracorporeal life support for refractory out-of-hospital cardiac arrest: Should we still fight for? A single-centre, 5-year experience.Int J Cardiol. 2016;204:70-6. doi: 10.1016/j.ijcard.2015.11.165.

37. Beppu K, Doi T, Hosokawa A, et al. Full neurological recovery, Cerebral Performance Category (CPC) 1, after 65-minute cardiac arrest using percutaneous cardiopulmonary system and therapeutic hypothermia. Int J Cardiol. 2013;168:4893-95. doi: 10.1016/j.ijcard.2013.07.024

38. Kirkham F. Cardiac arrest and post resuscitation of the brain. Eur J Paediatr Neurol. 2011;15:379-89. doi: 10.1016/j. ejpn.2011.04.009.

39. Mochizuki K, Imamura H, Iwashita T, Okamoto K. Neurological outcomes after extracorporeal cardiopulmonary resuscitation in patients with out-of-hospital cardiac arrest: a retrospective observational study in a rural tertiary care center. J Intensive Care. 2014;2:33. doi: 10.1186/2052-0492-2-33.

40. Yagi T, Nagao K, Sakatani K, et al. Changes of cerebral oxygen metabolism and hemodynamics during ECPR with hypothermia measured by near-infrared spectroscopy: a pilot study. Adv Exp Med Biol. 2013;789:121-8. doi: 10.1007/978-1-4614-74111_17.
41. Nagao K, Kikushima K, Watanabe K, et al. Early induction of hypothermia during cardiac arrest improves neurological outcomes in patients with out-of-hospital cardiac arrest who undergo emergency cardiopulmonary bypass and percutaneous coronary intervention. Circ J. 2010;74:77-85.

42. Fagnoul D, Combes A, De Backer D. Extracorporeal cardiopulmonary resuscitation. Curr Opin Crit Care. 2014;20:259-65. doi: 10.1097/MCC.0000000000000098.

43. Kim SJ, Jung JS, Park JH, Park JS, Hong YS, Lee SW. An optimal transition time to extracorporeal cardiopulmonary resuscitation for predicting good neurological outcome in patients with outof-hospital cardiac arrest: a propensity-matched study. Crit Care. 2014;18:535. doi: 10.1186/s13054-014-0535-8.

44. Benedek T, Jako B, Benedek I. Plaque Quantification by Coronary CT and Intravascular Ultrasound Identifies a Low CT Density Core as a marker of plaque instability in Acute Coronary Syndromes. International Heart J. 2014;55:22-8.

45. Farb A, Burke AP, Tang AL, et al. Coronary plaque erosion without rupture into a lipid core. A frequent cause of coronary thrombosis in sudden coronary death. Circulation. 1996; 93:1354.

46. Otani T, Sawano H, Oyama K, Morita M, Natsukawa T, Kai T. Resistance to conventional cardiopulmonary resuscitation in witnessed out-of-hospital cardiac arrest patients with shockable initial cardiac rhythm. J Cardiol. 2016;68:161-7. doi: 10.1016/j.jjcc.2015.08.020.

47. Lazzeri C, Valente S, Peris A, Gensini GF. Extracorporeal life support for out-of-hospital cardiac arrest: Part of a treatment bundle. Eur Heart J Acute Cardiovasc Care. 2015; pii:2048872615585517. DOI: 10.1177/2048872615585517 [Epub ahead of print].

48. Lazzeri C, Valente S, Peris A, Gensini GF. Extracorporeal life support treatment bundle for refractory cardiac arrest. Resuscitation. 2015;87:e5-e6. doi: 10.1016/j. resuscitation.2014.10.027.

49. Stub D, Bernard S, Pellegrino V, et al. Refractory cardiac arrest treated with mechanical CPR, hypothermia, ECMO and early reperfusion (the CHEER trial). Resuscitation. 2015;86:88-94. doi: 10.1016/j.resuscitation.2014.09.010.

50. Belohlavek J, Kucera K, Jarkovsky J, et al. Hyperinvasive approach to out-of hospital cardiac arrest using mechanical chest compression device, prehospital intraarrest cooling, extracorporeal life support and early invasive assessment compared to standard of care. A randomized parallel groups comparative study proposal. "Prague OHCA study". J Transl Med. 2012;10:163. doi: 10.1186/1479-5876-10-163.

51. Kagawa E, Dote $K$, Kato $M$, et al. Should we emergently revascularize occluded coronaries for cardiac arrest?: rapid-response extracorporeal membrane oxygenation and intra-arrest percutaneous coronary intervention. Circulation. 2012;126:1605-13. DOI: 10.1161/ CIRCULATIONAHA.111.067538.

52. Kagawa E, Dote K, Kihara Y. Percutaneous coronary intervention for postcardiac arrest syndrome. Curr Opin Cardiol. 
Available online at: www.jccm.ro

2013;28:689-94. doi: 10.1097/HCO.0b013e32836553b7.

53. Sakamoto T, Morimura N, Nagao K, et al. Extracorporeal cardiopulmonary resuscitation versus conventional cardiopulmonary resuscitation in adults with out-of-hospital cardiac arrest: a prospective observational study. Resuscitation. 2014;85:762-8. doi: 10.1016/j.resuscitation.2014.01.031.

54. Yamada T, Kitamura T, Hayakawa K, et al.Rationale, design, and profile of Comprehensive Registry of In-Hospital Intensive Care for OHCA Survival (CRITICAL) study in Osaka, Japan. J Intensive Care. 2016;4:10. doi: 10.1186/s40560-016-0128-5.

55. Rassaf T, Westenfeld R, Kurt M, Lichtenberg A, Kelm M. Rapid response ECLS after 140 min of refractory ventricular fibrillation following out-of-hospital cardiac arrest: feasibility as bridge to primary PCl. Resuscitation. 2014;85:e57-e59. doi: 10.1016/j. resuscitation.2013.12.029.

56. Beurtheret S, Mordant P, Paoletti X, et al.Emergency circulatory support in refractory cardiogenic shock patients in remote institutions: a pilot study (the cardiac-RESCUE program). Eur Heart J. 2013;34:112-20. doi: 10.1093/eurheartj/ehs081.

57. Benedek T, Dobreanu D. Current Concepts and New Trends in the Treatment of Cardiogenic Shock Complicating Acute Myocardial Infarction. J Crit Care Med. 2015;1:5-11. DOI: 10.1515/jccm-2015-0002

58. Bărcan A, Chițu M, Benedek E, et al. Predictors Of Mortality In Patients With ST-Segment Elevation Acute Myocardial Infarction And Resuscitated Out-Of-Hospital Cardiac Arrest. J Crit Care Med. 2016;2:22-30. DOI: 10.1515/jccm-2016-0001

59. Bajka B, Orzan M, Jakó B, Kovács I. Distance-related differences in critical times, protocol activation and mortality in a regional STEMI network. Journal of Cardiovascular Emergencies. 2016;2:1228. DOI: 10.1515/jce-2016-0019.

60. Peek GJ. Community extracorporeal life support for cardiac arrest-when should it be used? Resuscitation. 2011;82:1117. doi: 10.1016/j.resuscitation.2011.06.006.

61. Strapazzon G, Plankensteiner J, Mair P, et al. Prehospital management and outcome of avalanche patients with out-ofhospital cardiac arrest: a retrospective study in Tyrol, Austria. Eur J Emerg Med. 2016. DOI: 10.1097/MEJ.0000000000000390 [Epub ahead of print].

62. Hwang WS, Park JS, Kim SJ, Hong YS, Moon SW, Lee SW. A system-wide approach from the community to the hospital for improving neurologic outcomes in out-of-hospital cardiac arrest patients. Eur J Emerg Med. 2015. DOI: 10.1097/ MEJ.0000000000000313 [Epub ahead of print].

63. Avalli L, Mauri T, Citerio G, et al. New treatment bundles improve survival in out-of-hospital cardiac arrest patients: a historical comparison. Resuscitation. 2014;85:1240-4. doi: 10.1016/j.resuscitation.2014.06.014.

64. Grunau B, Reynolds J, Scheuermeyer F, et al. Relationship between Time-to-ROSC and Survival in Out-of-hospital Cardiac Arrest ECPR Candidates: When is the Best Time to Consider Transport to Hospital? Prehosp Emerg Care. 2016;20:615-22.
The Journal of Critical Care Medicine 2016;2(4) • 173 doi: 10.3109/10903127.2016.1149652.

65. Grunau B, Scheuermeyer FX, Stub D, et al. Potential Candidates for a Structured Canadian ECPR Program for Out-of-Hospital Cardiac Arrest. CJEM. 2016:1-8. DOI: 10.1017/cem.2016.8.

66. Sharma AS, Pijls RW, Weerwind PW, et al. Out-of-hospital cardiac arrest: the prospect of E-CPR in the Maastricht region. Neth Heart J. 2016;24:120-6. doi: 10.1007/s12471-015-07826.

67. Yannopoulos D, BartosJA, Martin C, etal.Minnesota Resuscitation Consortium's Advanced Perfusion and Reperfusion Cardiac Life Support Strategy for Out-of-Hospital Refractory Ventricular Fibrillation. J Am Heart Assoc. 2016;5:e003732. doi: 10.1161/ JAHA.116.003732.

68. Arima T, Nagata O, Sakaida K, et al. Relationship between duration of prehospital resuscitation and favorable prognosis in ventricular fibrillation. Am J Emerg Med. 2015;33:677-81. doi: 10.1016/j.ajem.2015.02.031.

69. Leick J, Liebetrau C, Szardien S, et al. Door-to-implantation time of extracorporeal life support systems predicts mortality in patients with out-of-hospital cardiac arrest. Clin Res Cardiol. 2013;102:661-9. doi: 10.1007/s00392-013-0580-3.

70. Spangenberg T, Meincke F, Brooks S, et al."Shock and Go?" extracorporeal cardio-pulmonary resuscitation in the goldenhour of ROSC. Catheter Cardiovasc Interv. 2016. doi: 10.1002/ ccd.26616 [Epub ahead of print].

71. Johnson NJ, Acker $\mathrm{M}, \mathrm{Hsu} \mathrm{CH}$, et al. Extracorporeal life support as rescue strategy for out-of-hospital and emergency department cardiac arrest. Resuscitation. 2014;85:1527-32. doi: 10.1016/j.resuscitation.2014.08.028.

72. Rubertsson S. Update on mechanical cardiopulmonary resuscitation devices. Curr Opin Crit Care. 2016;22:225-9. doi: 10.1097/MCC.0000000000000302.

73. Menegazzi JJ, Salcido DD, Housler GJ, Logue ES. Feasibility of initiating extracorporeal life support during mechanical chest compression CPR: a porcine pilot study. Resuscitation. 2012;83:130-3. doi: 10.1016/j.resuscitation.2011.07.030

74. Agostinucci JM, Ruscev M, Galinski M, et al. Out-of-hospital use of an automated chest compression device: facilitating access to extracorporeal life support or non-heart-beating organ procurement. Am J Emerg Med. 2011;29:1169-72. doi: 10.1016/j.ajem.2010.06.029.

75. Ogawa Y, Shiozaki T, Hirose T, et al. Load-distributing-band cardiopulmonary resuscitation for out-of-hospital cardiac arrest increases regional cerebral oxygenation: a single-center prospective pilot study. Scand J Trauma Resusc Emerg Med. 2015;23:99. doi: 10.1186/s13049-015-0182-3.

76. Couper K, Smyth M, Perkins GD. Mechanical devices for chest compression: to use or not to use? Curr Opin Crit Care. 2015;21:188-94. doi: 10.1097/MCC.0000000000000200.

77. Topjian AA, Naim MY, Nadkarni V. To cool or not to cool during cardiopulmonary resuscitation. World J Pediatr Congenit Heart Surg. 2012;3:54-7. doi: 10.1177/2150135111418256.

78. Kim SJ, Kim HJ, Lee HY, Ahn HS, LeeSW. Comparing extracorporeal 
174 - The Journal of Critical Care Medicine 2016;2(4)

cardiopulmonary resuscitation with conventional cardiopulmonary resuscitation: A meta-analysis. Resuscitation. 2016;103:106-16. doi: 10.1016/j.resuscitation.2016.01.019.

79. Haneya A, Philipp A, Diez C, et al. A 5-year experience with cardiopulmonary resuscitation using extracorporeal life support in non-postcardiotomy patients with cardiac arrest. Resuscitation. 2012;83:1331-7. doi: 10.1016/j. resuscitation.2012.07.009.

80. Jung $C$, Janssen $K$, Kaluza $M$, et al. Outcome predictors in cardiopulmonary resuscitation facilitated by extracorporeal membrane oxygenation. Clin Res Cardiol. 2016;105:196-205. doi: 10.1007/s00392-015-0906-4.
Available online at: www.jccm.ro

81. Anselmi A, Flécher E. Extracorporeal cardiopulmonary resuscitation for out-of-hospital refractory cardiac arrest: A word of caution. J Thorac Cardiovasc Surg. 2016;151:1217-8. doi: 10.1016/j.jtcvs.2015.11.033.

82. Anselmi A, Flécher $E$, Corbineau $H$, et al. Survival and quality of life after extracorporeal life support for refractory cardiac arrest: A case series. J Thorac Cardiovasc Surg. 2015;150:94754. doi: 10.1016/j.jtcvs.2015.05.070.

83. Flécher $E$, Anselmi A, Corbineau $H$, et al. Current aspects of extracorporeal membrane oxygenation in a tertiary referral centre: determinants of survival at follow-up. Eur J Cardiothorac Surg. 2014;46:665-71. doi: 10.1093/ejcts/ezu029. 